\section{ERS School Course Basic principles of clinical exercise testing}

\section{Clinical exercise testing}

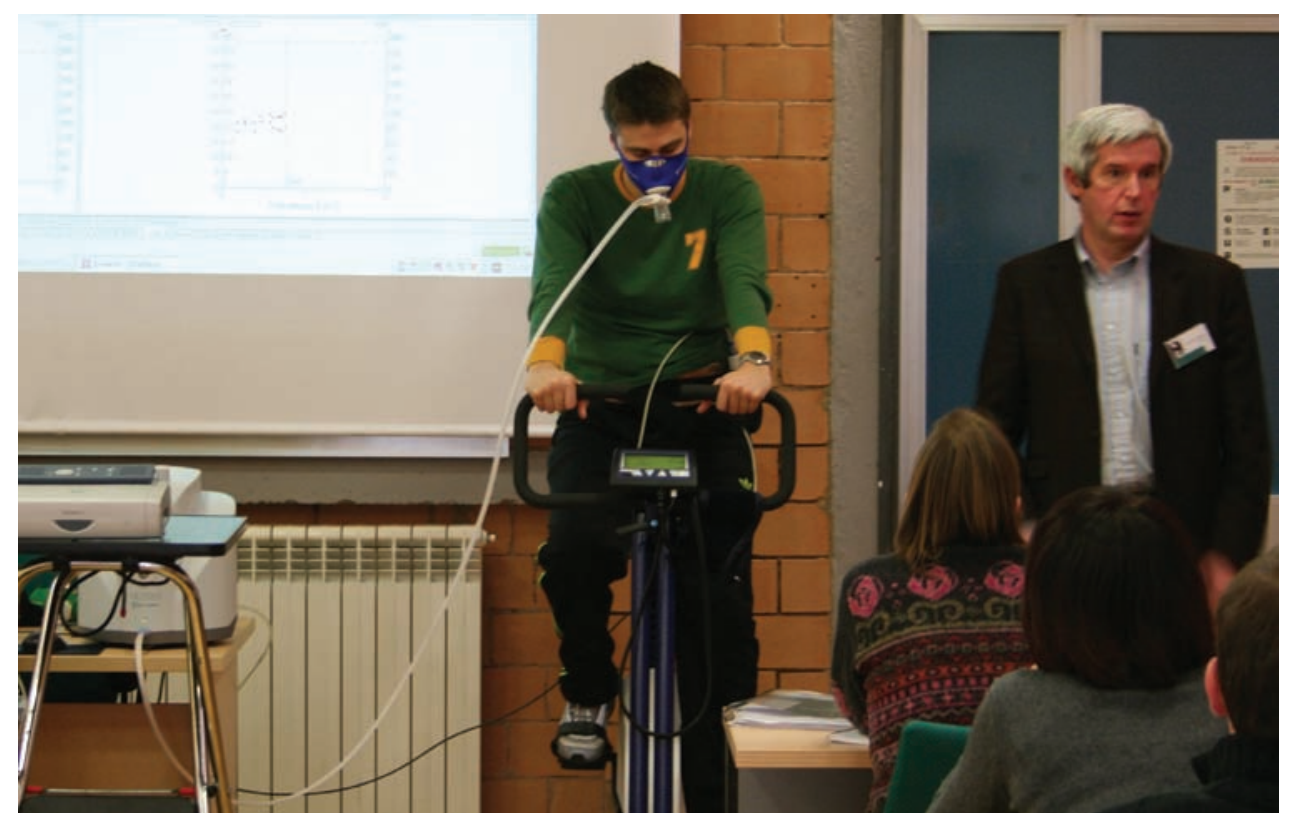

\section{Educational aims}

1 To provide a comprehensive introduction to the physiological basis of integrative exercise testing.

I To identify the indications and the protocols used in clinical settings.

1 To discuss the clinical applications of exercise testing with special reference to lung and heart diseases.

1 To understand the utility of exercise testing in the prescription and evaluation of therapeutic interventions and in rehabilitation.

\section{Summary}

Exercise intolerance is the hallmark of pulmonary and cardiac diseases. However, measurements of lung and cardiac function obtained at rest (e.g. forced expiratory volume in one second ( $\mathrm{FEV} 1)$, pulmonary diffusing capacity of the lung for carbon monoxide $(D L, C O)$, left ventricular ejection fraction (EF)) are poorly predictive of the degree of exercise intolerance. Therefore, it is necessary to directly assess an individual's exercise intolerance and, where possible, establish its cause(s). The "gold standard" approach for this is cardiopulmonary exercise testing (CPET). CPET has also proven to be very useful in the following applications: prognostic evaluation of patients with chronic lung and heart diseases, such as chronic obstructive pulmonary diseases (COPD), interstitial lung diseases (ILD), cystic fibrosis (CF) and chronic heart failure (CHF); and evaluating the effects of interventions such as drug therapy, oxygen and heliox supplementation during exercise, and pulmonary rehabilitation based on exercise training.
This article is adapted from the ERS School Course on Basic principles of clinical exercise testing organised in Rome, March 2-4, 2006. Original slides, web casts and additional material can be found at www.ers-education.org.
P. Palange 1

S.A. Ward ${ }^{2}$

B.J. Whipp 2

${ }^{1}$ Dipartimento di Medicina Clinica, Università "La Sapienza di Roma", Rome, Italy. ${ }^{2}$ Institute of Membrane and Systems Biology, University of Leeds, Leeds, UK.

Correspondence:

P. Palange

Dipartimento di Medicina Clinica V. le Università 37

185 Rome

Italy

Fax: 39064940421

E-mail:

Paolo.Palange@uniroma1.it 
Figure 1

Factors limiting exercise tolerance.

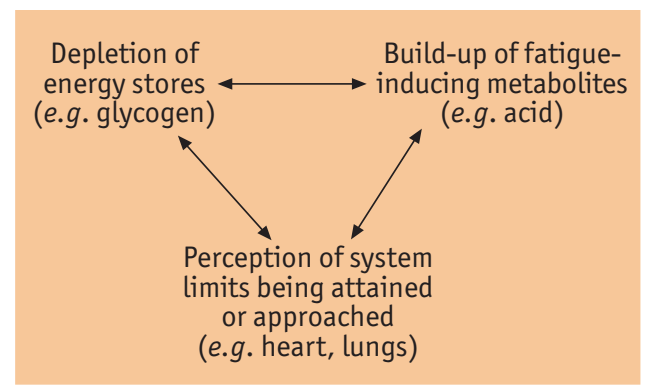

\section{Pathophysiology of exercise limitation}

Exercise intolerance results when a subject is unable to sustain the required work rate (WR) sufficiently long enough for the successful completion of the task in question; it is, therefore, task specific. Exercise performed with a large muscle mass (e.g. the legs) can be sustained longer than that performed with a smaller muscle mass (e.g. the arms); tasks partitioned into work-rest cycles can be sustained for longer than continuous periods of exercise, as the intensity is reduced despite the same WR being performed. The factors typically limiting exercise tolerance are illustrated in figure 1.

In extreme cases of patients with COPD, for example, exercise intolerance can limit subjects to a mild domiciliary routine, and is predominantly (although, interestingly, not exclusively) a consequence of shortness of breath or dyspnoea. This is typically associated with increased ventilatory or airflow demands that encroach upon the reduced limits of respiratory-mechanical performance.

\section{Purposes of exercise testing}

The main purposes of exercise testing are listed in table 1.

The goal of exercise testing is to stress the physiological systems contributing to the exercise intolerance to a level at which an abnormality becomes discernible from the magnitude

\section{Table 1 Main purposes of exercise testing}

\section{Establishing the:}

Limits of system function

Effective operating range

Normalcy with respect to an appropriate reference population

Normalcy with respect to other physiological functions

Means of simulating activity

Frame of reference for change

Means of "triggering" abnormality and, often most importantly,

Identifying the cause(s) of exercise intolerance and/or profile of appropriately selected variables. Most modern clinical exercise testing laboratories now have the ability to compute and display a wide range of system response variables on a breath-by-breath basis. Assessing the normalcy, or otherwise, of the system responses to the exercise requires the investigator to select, and appropriately display, the cluster of response variables that are themselves reflective of the particular system(s) behaviour. The interpretation of the results is then based on two interrelated perspectives: discriminating a magnitude or pattern of deviation from the normal response (of the age-, sex- and activity-matched standard subject); and matching the magnitude or pattern of abnormality with that characteristic of particular impairments of system function.

The challenge of exercise testing is, therefore, to devise or, more usually, utilise a previously devised test format that is most discriminating with respect to the particular question under consideration. A range of such tests is available to the investigator. As the goal of exercise testing is to evaluate the functioning of the organ systems contributing to the exercise intolerance, testing generally involves exercising large muscle groups, usually the lower extremity muscles, although arm or other exercise strategies can be effectively utilised for particular purposes.

\section{Exercise tolerance and exercise intensity}

While the tolerable duration of a given WR is known to depend upon the intensity of the exercise being performed, there is, to date, no generally agreed method for characterising work intensity. Two widely used procedures fail to meet the demands of critical scrutiny in this regard: the "met" increment and the "percentage" of the maximal oxygen uptake $\left(\mathrm{VO}_{2}, \mathrm{max}\right)$. The onset of the metabolic (lactic) acidaemia of exercise (i.e. the lactate threshold $(\theta \mathrm{L})$ ) does not occur at a common met increment in different individuals. Consequently, different subjects at the same met level can have markedly different degrees of metabolic acidaemia. Similarly, while in normal individuals $\theta \mathrm{L}$ occurs at $~ 50 \%$ of the $\mathrm{VO}_{2}$, max on average, the on average distribution is very large, with the normal range extending from $\sim 35 \%$ to $\geq 80 \%$. Consequently, if the exercise intensity is assigned to a particular percentage of $\mathrm{VO}_{2}$, max $($ e.g. $70 \%)$, then one subject 

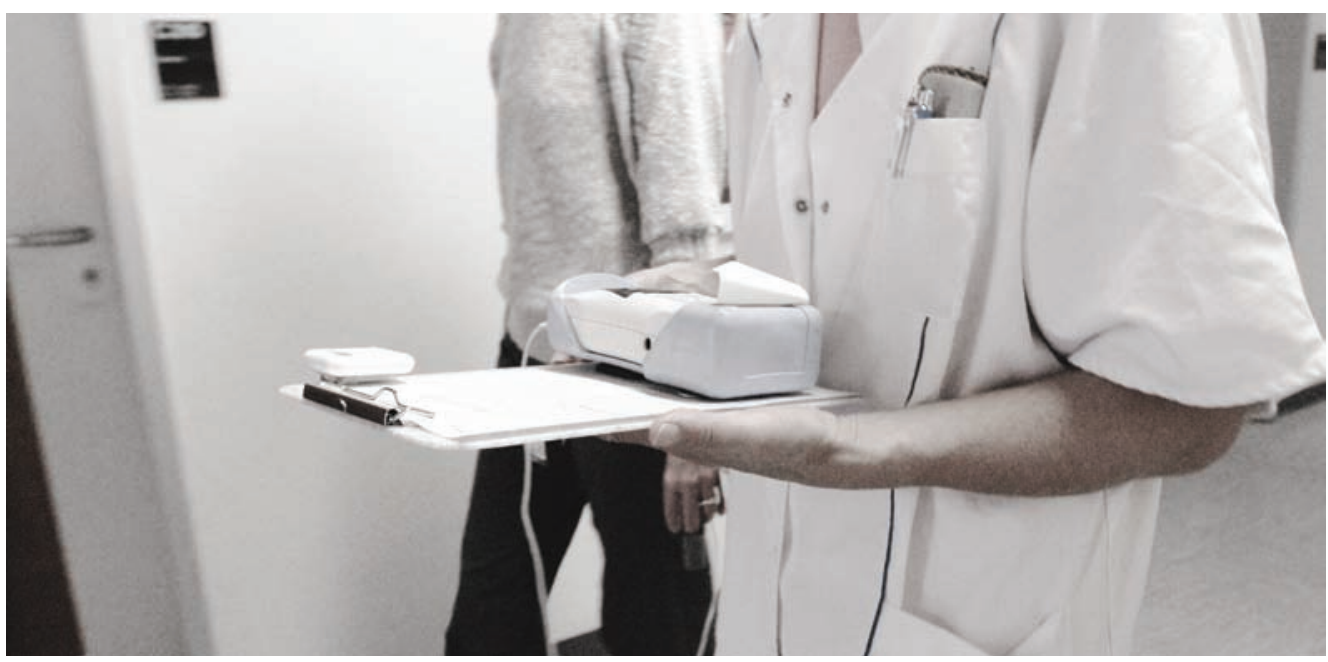

could be exercising at a sub-threshold work rate and be "comfortable", whereas another could be exhausted at $\mathrm{VO}_{2}$, max.

Often, however, patients may not be able to attain a $\mathrm{VO}_{2}$, max in the conventional sense (or the investigator may not wish to push them to these levels) because of limitation by some system-related perception (e.g. angina, dyspnoea, claudicating pain). However, as the estimation of $\theta L$ does not require such efforts, it provides a useful index of the onset of an exercise-induced metabolic acidaemia. However, its discriminability under "complicating" conditions, such as chronic hyperventilation syndromes, progressive exercise-induced hypoxaemia or impaired peripheral chemosensitivity with an associated high airway resistance, remains to be established.

\section{Exercise testing and prognostic evaluation}

There is now a growing body of evidence for exercise tolerance being a good predictor of mortality in healthy adults, across a wide age range, and also for many cardiovascular and pulmonary diseases. Perhaps the best known example where a comprehensive cluster of CPET-based prognostic variables has been established is that of CHF. This recognition is one of the driving forces for exercise testing becoming an essential component of the assessment procedures in cardiovascular and pulmonary disease. In the case of cardiovascular disease, for example, both the current classifications of disease severity and the current guidelines for heart transplantation (figure 2) now incorporate considerations of CPET-derived indices, such as $\mathrm{VO}_{2}$, peak, as well as $\mathrm{VO}_{2}$ at $\mathrm{\theta L}$, the slope of the minute ventilationto- $\mathrm{CO}_{2}$ output relationship (i.e. $\Delta V \mathrm{E} / \Delta V \mathrm{CO}_{2}$ ) over the appropriate linear response range, and also the absolute $V \mathrm{E} / \mathrm{VCO}_{2}$ value at $\mathrm{\theta L}$.

In contrast, the situation for pulmonary disthat $\mathrm{VO}_{2}$,peak is valuable in the prognostic evaluation of patients with end-stage COPD and CF, for example, and also in risk stratification. Interestingly, due to their practical utility, fieldbased exercise tests (e.g. six-minute walking test (6MWT), shuttle walking test) are growing in popularity as indices of exercise capacity in patients with lung disease, with outcomes being assessed via indices such as the distance covered during the test, heart rate and the degree of arterial $\mathrm{O}_{2}$ desaturation. The $6 \mathrm{MWT}$ is frequently preferred, since the performance of a conventional maximal incremental exercise test may be particularly challenging for patients with severe lung disease, particularly in the context of transplant evaluation. Furthermore, it has been reported that 6MWT distance is a good predictor of $\mathrm{VO}_{2}$, peak in patients with end-stage lung diseases, especially if the following factors are taken into account: age, weight, forced vital capacity (FVC), FEV 1 and $D L, C O$. Also, there is an increased risk of death in patients with $\mathrm{CF}$ ease is less clear. Nonetheless, it is now evident
Educational questions

Are the following statements true or false?

1. Exercise tolerance is a good predictor of mortality in healthy subjects and patients with chronic diseases (e.g. CHF, COPD).

2. In patients with COPD and $\mathrm{CHF}$, exercise tolerance can be predicted by physiological measurement obtained at rest.

3. CPET is considered the gold standard to assess the degree and the cause(s) of exercise intolerance.

4. Exercise testing is increasingly utilised for the prognostic evaluation of patients with chronic cardiac and pulmonary diseases.

5. High-intensity constant work rate protocols with measurement of "endurance time" (time to symptom limitation (tLIM)) are very useful in the evaluation of therapeutic interventions (e.g. bronchodilators, oxygen/ heliox, rehabilitation).

\section{Figure 2}

Selection of patients for heart transplantation in the current era of heart failure therapy.

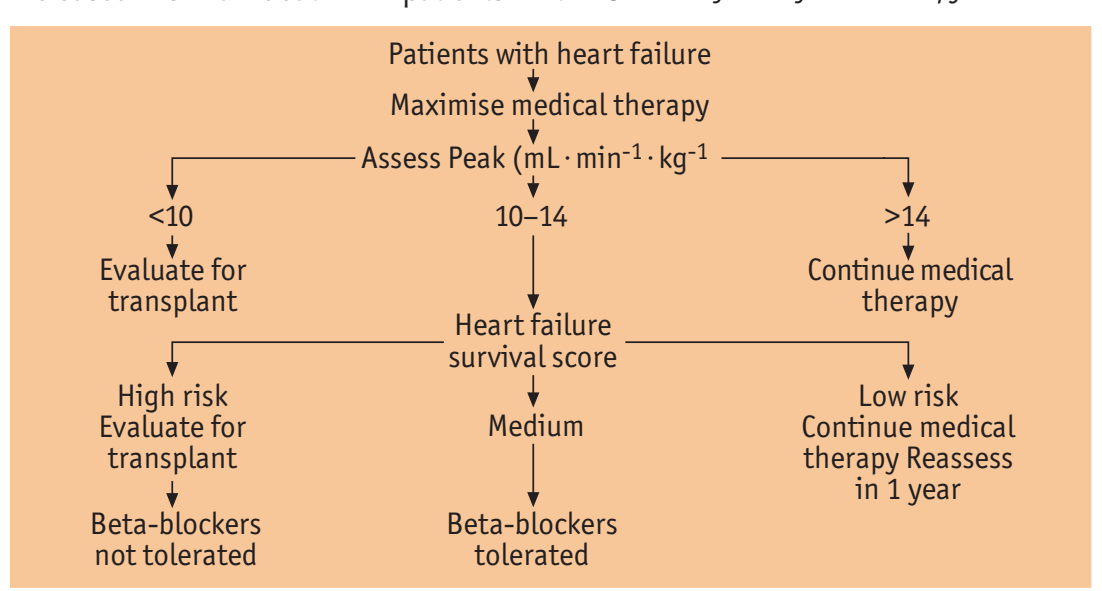




\section{What is the "best" measure of improvement in exercise tolerance?}

Improvements in exercise tolerance in patients with cardiorespiratory diseases can be assessed via a range of exercise protocols and measures. The "gold standard" approach for assessment of exercise tolerance remains the laboratory symptom-limited incremental test (cycle ergometer or treadmill), with measurement of $\mathrm{VO}_{2}$,peak and related indices. However, when interventional change is being assessed, this test is often supplemented by a high-intensity constant-load "endurance" test performed to the limit of tolerance, as the time to symptom limitation (tum) can provide a more sensitive index of improved exercise tolerance. What are termed "field" tests, with measurement of maximal distance walked, have been shown to be useful in this context. This is particulary well exemplified for COPD, in which there is a growing body of evidence that high-intensity constant-load endurance protocols with measurement of $t \mathrm{LIM}$, supplemented by the measurement of symptoms (e.g. dyspnoea and leg fatigue) and pertinent CPET variables at "isotime", are superior to other indices of exercise tolerance (e.g. $\mathrm{VO}_{2}$, peak on maximal incremental test, distance on 6MWT) in evaluating the efficacy of therapeutic interventions.

While measurement of effort-dependent measures of exercise tolerance (e.g. $\mathrm{VO}_{2}$, peak, tuIM) is clearly important in assessing interventional change, it is also useful to monitor the degree of change in the physiological response (both in terms of response amplitude and its profile of development) to identical incremental and/or constant-load challenges. It is important to emphasise "task specificity" in this regard. For example, interventions targeted at improving endurance of the upper extremity muscles might be expected to be expressed as an increased endurance time during arm ergometry, but not during cycle ergometry. Finally, when clinical trials are being undertaken or for individualised patient care, knowledge of the minimally clinically important difference for key indices of exercise tolerance is important.

awaiting lung transplantation who evidence a high degree of breathing reserve (i.e. $\mathrm{VE} /$ maximal voluntary ventilation) elevation at $\theta \mathrm{L}$.

\section{Evaluation of therapeutic interventions}

Based on the considerable evidence supporting the superiority of exercise testing over resting measures, such as FEV1 and EF, in demonstrating responses to a range of interventions, exercise tolerance is now an important outcome measure for patients with a range of chronic cardiorespiratory diseases (e.g. COPD, CHF). This applies, for example, to exercisebased rehabilitation (both in terms of short and longer-term benefits), drug therapy, supplemental oxygen therapy, heliox breathing, nutritional therapy, hormonal therapy and also surgical interventions (e.g. lung volume reduction surgery, lung transplantation).

\section{Recommendations for clinical practice}

There is a now an appreciable body of evidence, covering a range of pulmonary and cardiovascular diseases, which illustrates the benefits of employing CPET. For example, in addition to the assessment of exercise intolerance, the complement of physiological responses measured during CPET has been shown to be reproducible in the short term, and in the longer term can reflect disease progression. Indices of exercise tolerance and related CPET variables have been shown to be significantly better predictors of prognosis than classical measures of resting pulmonary or cardiac function. CPET has proven valuable in evaluating both prognosis and the effects of therapeutic interventions in adults with chronic lung disease (COPD, ILD, primary pulmonary hypertension), in adults and children with CF and exercise-induced bronchospasm, in adults with $\mathrm{CHF}$, and also children and adolescents with congenital heart disease. In addition, CPETbased measures are useful in clinical trials for evaluating interventions in patients with pulmonary and cardiac diseases. Indeed, this is now the main indication for exercise testing in these patient groups. CPET variables are also useful in the individual prescription of pulmonary rehabilitation. Finally, endurance performance, assessed by tLIM on high-intensity constant-load protocols, is superior to conventional indices of resting lung and cardiac function in the evaluation of the effects of interventions. 


\section{Suggested further reading}

ATS/ACCP Statement on Cardiopulmonary Exercise Testing. Am J Respir Crit Care Med 2003; 167: 211-277.

Clinical exercise testing with reference to lung diseases: indications, standardization and interpretation strategies. ERS Task Force on Standardization of Clinical Exercise Testing. European Respiratory Society. Eur Respir J 1997; 10: 2662-2689.

Roca J, Whipp BJ, eds. Clinical Exercise Testing. Monograph 6. Sheffield, European Respiratory Society Journals, 1997.

Wasserman K, Hansen JE, Sue DY, Stringer W, Whipp BJ. Principles of Exercise Testing and Interpretation. 4th Edn. Philadelphia, Lea \& Febiger, 2004.

Palange P, Carlone S, Forte S, Galassetti P, Serra P. Cardiopulmonary exercise testing in the evaluation of patients with ventilatory vs circulatory causes of reduced exercise tolerance. Chest 1994; 105: 1122-1126.

O'Donnell DE, Revill SM, Webb KA. Dynamic hyperinflation and exercise intolerance in chronic obstructive pulmonary disease. Am J Respir Crit Care Med 2001; 164: 770-777.

Gitt $A K$, Wasserman K, Kilkowski C, et al. Exercise anaerobic threshold and ventilatory efficiency identify heart failure patients for high risk of early death. Circulation 2002; 106: 3079-3084.

Oga T, Nishimura K, Tsukino M, Sato S, Hajiro T. Analysis of the factors related to mortality in chronic obstructive pulmonary disease: role of exercise capacity and health status. Am J Respir Crit Care Med 2003; 167: 544-549.

Arena R, Myers J, Aslam SS, Varughese EB, Peberdy MA. Peak V'O2 and V'E/V'CO2 slope in patients with heart failure: a prognostic comparison. Am Heart J 2004; 147: 354-360.

Neder JA, Jones PW, Nery LE, Whipp BJ. Determinants of the exercise endurance capacity in patients with chronic obstructive pulmonary disease. The power-duration relationship. Am J Respir Crit Care Med 2000; 162: 497-504.

Oga T, Nishimura K, Tsukino M, Hajiro T, Ikeda A, Izumi T. The effects of oxitropium bromide on exercise performance in patients with stable chronic obstructive pulmonary disease. A comparison of three different exercise tests. Am J Respir Crit Care Med 2000; 161: 1897-1901.

O'Donnell DE, Fluge T, Gerken F, et al. Effects of tiotropium on lung hyperinflation, dyspnoea and exercise tolerance in COPD. Eur Respir J 2004; 23: 832-840.

Palange $P$, Valli $G$, Onorati $P$, et al. Effect of heliox on lung dynamic hyperinflation, dyspnea, and exercise endurance capacity in COPD patients. J Appl Physiol 2004; 97: 1637-1642.

Emtner M, Porszasz J, Burns M, Somfay A, Casaburi R. Benefits of supplemental oxygen in exercise training in nonhypoxemic chronic obstructive pulmonary disease patients. Am J Respir Crit Care Med 2003; 168: 1034-1042.

Porszasz J, Emtner M, Goto S, Somfay A, Whipp BJ, Casaburi R. Exercise training decreases ventilatory requirements and exercise-induced hyperinflation at submaximal intensities in patients with COPD. Chest 2005; 128: 2025-2034.

Palange P, Ward SA, Carlsen KH, et al. Recommendations on the use of exercise testing in clinical practice. Eur Respir J 2007; (In press).
A report of the ERS Task Force on "Clinical Exercise Testing", which includes evidence-based indications for exercise testing in clinical practice, will be published shortly in the European Respiratory Journal.

\section{Suggested answers}

1. True.

2. False.

3. True.

4. True.

5. False. 